2 - 43 months). There was a mean decrease in opening pressure (OP) on LP from pre- to post-VSS of $9.1 \mathrm{~cm} \mathrm{H} 2 \mathrm{O}$ (median $9.5 \mathrm{~cm} \mathrm{H} 2 \mathrm{O}$ ). Of the 44 patients, 18 (22.2\%) underwent repeat angiogram to evaluate for candidacy for repeat stenting, of which $5(27.8 \%, 6.2 \%$ of total $)$ patients underwent a second stenting procedure. Eighteen of the $44(41.9 \%$, $22.2 \%$ of total) patients underwent a subsequent CSF shunting procedure at a mean of 7.1 months (median 5.7 months) following VSS. Overall, a total of 21 (25.9\%) patients underwent further surgical intervention following VSS. Forty-six patients were administered quality of life (WHOQOL-BREF) and symptom severity questionnaires (HIT-6) at initial consultation and each subsequent visit. There was an overall increase in quality of life scores with mean pre-stenting and last followup (post-VSS) scores of 61.2 (SEM 2.5) and 71.2 (SEM 3.9), respectively. There was an overall decrease in HIT-6 scores with mean pre-stenting and last follow-up (post-VSS) scores of 62.7 (SEM 1.7) and 55.8 (SEM 2.9), respectively.

Conclusions VSS is an effective treatment for venous sinus stenosis in IIH, however, this study found higher rates of symptomatic recurrence and need for further surgical intervention than has previously been reported in the literature. Recurrence of symptoms occurs at a median of 7 months even though OP remain lower at follow-up LP suggestive of a re-equilibration phenomenon.

Disclosures R. Garner: None. J. Aldridge: None. S. Wolfe: None. K. Fargen: None.

\section{E-004 SEX-BASED DIFFERENCE IN THE UTILIZATION OF ENDOVASCULAR THERAPY IN CEREBRAL SINUS THROMBOSIS: THE USA BASED STUDY}

${ }^{1} \mathrm{~S}$ Patel", ${ }^{2} \mathrm{~N}$ Desai, ${ }^{1} \mathrm{M}$ Pervez. ${ }^{1}$ Neurology, University of Connecticut/Hartford hospital, Hartford, $C T$; ${ }^{2}$ Neurology, NYU langone medical center, NY, NY

\subsection{6/neurintsurg-2020-SNIS.40}

Introduction Cerebral sinus thrombosis (CVT) is an overall uncommon cause of cerebral venous infarction that accounts for approximately $0.5-1 \%$ of all strokes. The initial treatment for CVT is systemic anticoagulation, which has been associated with improved neurological outcomes in small randomized studies. However, with anticoagulation alone, large and extensive thrombi are unlikely to dissolve, and thus approximately one-third of patients with severe presentation have a high risk of incomplete recovery. Endovascular intervention is an alternative option for patients with neurological deterioration despite the use of anticoagulation or with the development of new or worsening intracerebral hemorrhage (ICH) on anticoagulation. Current endovascular techniques include direct catheter thrombolysis, balloon-assisted thrombectomy, rheolytic catheter thrombectomy, aspiration thrombectomy, and stent retriever thrombectomy. Prior studies have reported a higher risk of mortality and poor outcomes in the EVT group. However, there is a lack of contemporary data regarding sex-based differences in outcomes. The goal of our study was to understand the sex-based trend of EVT utilization and differences in outcomes following CVT.

Methods We used the Nationwide Inpatient Sample database from the year 2005-2015 to identify CVT hospitalizations who treated with EVT using ICD-9 CM code. To make the data nationally representative, weights were applied as per NIS recommendations. Chi-square test and student's T-test
Abstract E-004 Table 1 Outcomes of Cerebral Sinus Thrombosis Hospitalizations in the US (2005-2015)

\begin{tabular}{|c|c|c|c|c|c|}
\hline & $\begin{array}{l}\text { Female } \\
\text { (N) }\end{array}$ & $\begin{array}{l}\text { Male } \\
(\mathrm{N})\end{array}$ & $\begin{array}{l}\text { Odds } \\
\text { ratios }\end{array}$ & $\begin{array}{l}\text { Lower limits- } \\
\text { Upper limits }\end{array}$ & $\begin{array}{l}\text { P- } \\
\text { value }\end{array}$ \\
\hline \multicolumn{6}{|l|}{ Discharge to home } \\
\hline $\begin{array}{l}\text { Medically treated group } \\
(\mathrm{N}=44,473)\end{array}$ & 30,970 & 13,503 & & & \\
\hline $\begin{array}{l}\text { Univariate model (female } \\
\text { vs. male) }\end{array}$ & & & 1.82 & $1.66-2.00$ & $<0.001$ \\
\hline $\begin{array}{l}\text { Multivariate model (female } \\
\text { vs. male) }\end{array}$ & & & 1.03 & $0.91-1.16$ & 0.69 \\
\hline $\begin{array}{l}\text { EVT treated group } \\
(\mathrm{N}=2,065)\end{array}$ & 1,384 & 681 & & & \\
\hline $\begin{array}{l}\text { Univariate model (female } \\
\text { vs. male) }\end{array}$ & & & 1.02 & $0.68-1.51$ & 0.94 \\
\hline $\begin{array}{l}\text { Multivariate model (female } \\
\text { vs. male) }\end{array}$ & & & 0.70 & $0.40-1.24$ & 0.22 \\
\hline In-hospital mortality & & & & & \\
\hline $\begin{array}{l}\text { Medically treated group } \\
(\mathrm{N}=44,473)\end{array}$ & 30,970 & 13,503 & & & \\
\hline $\begin{array}{l}\text { Univariate model (female } \\
\text { vs. male) }\end{array}$ & & & 0.45 & $0.35-0.57$ & $<0.001$ \\
\hline $\begin{array}{l}\text { Multivariate model (female } \\
\text { vs. male) }\end{array}$ & & & 0.78 & $0.57-1.06$ & 0.11 \\
\hline $\begin{array}{l}\text { EVT treated group } \\
(\mathrm{N}=2,065)\end{array}$ & 1,384 & 681 & & & \\
\hline $\begin{array}{l}\text { Univariate model (female } \\
\text { vs. male) }\end{array}$ & & & 1.64 & $0.83-3.24$ & 0.15 \\
\hline $\begin{array}{l}\text { Multivariate model (female } \\
\text { vs. male) }\end{array}$ & & & 1.60 & $0.62-4.11$ & 0.33 \\
\hline
\end{tabular}

-Multivariate model: Patient-level characteristics including Age, gender, Charlson comorbidity index, race, hematological conditions, CNS infections, brain tumors, any cancers, inflammatory conditions, traumatic brain injuries, DM, hypertension, drug abuse, smoking, EtOH abuse, seizures disorders, coma, cerebral edema, hydrocephalus, migraine, intracranial hemorrhage, ischemic stroke, ventriculostomy, invasive mechanical ventilation, tracheostomy and PEG tube.

-Hematological conditions, including sickle cell disorders, coagulation, hemorrhagic disorders, polycythemia, hypersplenism, and diseases of the spleen.

-Systemic inflammatory disease which includes systemic lupus erythematosus, rheumatoid disorders and other related conditions, and other connective tissue disorders.

-CNS infections include meningitis, encephalitis, other CNS infection and poliomyelitis -Charlson comorbidity index: The severity of co-morbid conditions was defined using the Charlson-Deyo Co-morbidity Index (CCI). $\mathrm{CCl}$ is an established measure to quantify the burden co-morbid conditions; the scores range from 0 to 33 with a higher score indicating a greater burden of concomitant diseases. Derived from: Roffman CE, Buchanan J, Allison GT. Charlson Comorbidities Index. Journal of physiotherapy. 2016;62(3):171.

were used for categorical and continuous variables to analyze descriptive data, respectively. A multivariate logistic regression model was performed on outcomes variables (discharge to home and in-hospital mortality) to avoid confounding effects.

Result A total of 46,537 CVT were extracted from the years 2005-2015. Among them, 2,065 treated with EVT (female $\mathrm{N}=1,384$ and male $\mathrm{N}=681$ ). The mean age of the EVT treated group was male $48.7 \pm 1.38$ vs. female $43.0 \pm 1.05$ $(\mathrm{p}=0.002)$. While in medically treated group mean age was male $49.5 \pm 0.38$ vs. female $40.6 \pm 0.26 \quad(p<0.001)$. After adjusting comorbidities, multivariable models did not show a statistical difference in mortality and home discharge among medically, and EVT treated gender groups (table). The overall trend of EVT utilization was increased from 29 cases in 2005 to 57 cases in 2015 per 1000 CVT hospitalization (trend $\mathrm{p}=0.04$ ). EVT in female group trended up (26 cases in 2005 to 57 cases in $2015 ; \mathrm{p}=0.04)$ while in male, EVT cases also 
trending up; however, without attaining statistical significance $(\mathrm{p}=0.45)$.

Conclusion Our nationally represented sample showed no difference in outcomes in medically, and EVT treated groups among gender despite prior literature suggesting poor outcomes in the EVT group. Patient selection for EVT should be individualized based on patient presentation. Our analysis showed CVT treated with EVT hospitalization is trending up in the US.

Disclosures S. Patel: None. N. Desai: None. M. Pervez: None.

\section{E-005 NORMALIZATION OF ELEVATED IDIOPATHIC INTRACRANIAL VENOUS PRESSURES AFTER MANOMETRY AND HIGH-VOLUME LUMBAR PUNCTURE IN A PATIENT WITH PSEUDOTUMOR CEREBRI}

${ }^{1} \mathrm{M}$ Brown*, ${ }^{2} \mathrm{~T}$ Wolfe. ${ }^{1}$ Radiology, Aurora St. Luke's Medical Center, Milwaukee, WI; ${ }^{2}$ Neuro-Interventional Radiology, Aurora St. Luke's Medical Center, Milwaukee, WI

10.1136/neurintsurg-2020-SNIS.41

Treatment of idiopathic intracranial hypertension (IIH), also known as pseudotumor cerebri, generally prioritizes alleviating headaches and preserving vision. If medical management is unsuccessful, possible surgical treatment options depending on symptom severity include serial lumbar punctures, venous sinus stenting, optic nerve sheath fenestration (ONSF), and cerebrospinal fluid (CSF) shunting. Venous sinus stenting has remained controversial as cerebral vein stenosis may not be a primary cause.

In our case, we present a 38-year-old female with known pseudotumor cerebri who underwent a diagnostic cerebral angiogram with manometry and high-volume lumbar puncture. Pre-lumbar puncture manometry demonstrated venous pressures throughout the left and right transverse sinuses and distal superior sagittal sinus ranging from $25-30 \mathrm{mmHg}$ with associated bilateral transverse-sigmoid junction stenoses. The patient was then placed in the lateral decubitus position and a lumber puncture at L2-3 was performed. Opening pressure was measured at $29 \mathrm{~cm} \mathrm{H} \mathrm{H}_{2} \mathrm{O}, 30 \mathrm{~mL}$ of clear CSF was removed, and closing pressure was measured at $8.5 \mathrm{~cm} \mathrm{H}_{2} \mathrm{O}$. Post-lumbar puncture manometry demonstrated normalization of respective venous pressures ranging from $8-12 \mathrm{mmHg}$ with resolution of associated bilateral transverse-sigmoid junction stenoses and improved venous sinus calibers. Following the procedure, the patient reported resolution of headache.

These results indicate that cerebral venous stenoses in the setting of IIH may be a secondary phenomenon. Thus, patients may benefit from CSF shunting as a primary surgical treatment option. In order to establish treatment efficacy, future studies could evaluate for stenosis and pressure gradient recurrence after CSF shunting.

Disclosures M. Brown: None. T. Wolfe: None.

\section{E-006 TUNING OF INNOVATIVE IN-VITRO MODEL MATERIALS TO MIMIC TISSUE PROPERTIES}

${ }^{1} \mathrm{~N}$ Norris ${ }^{*},{ }^{2} \mathrm{Smith},{ }^{2} \mathrm{C}$ Settanni, ${ }^{2} \mathrm{~W}$ Merritt, ${ }^{1,2} \mathrm{~T}$ Becker. ${ }^{1}$ Aneuvas Technologies Inc., Flagstaff, $A Z_{;}{ }^{2}$ Northern Arizona University, Flagstaff, $A Z$

10.1136/neurintsurg-2020-SNIS.42

Introduction/Purpose In-vitro models help test new medical devices for use in numerous cardiovascular and neurovascular

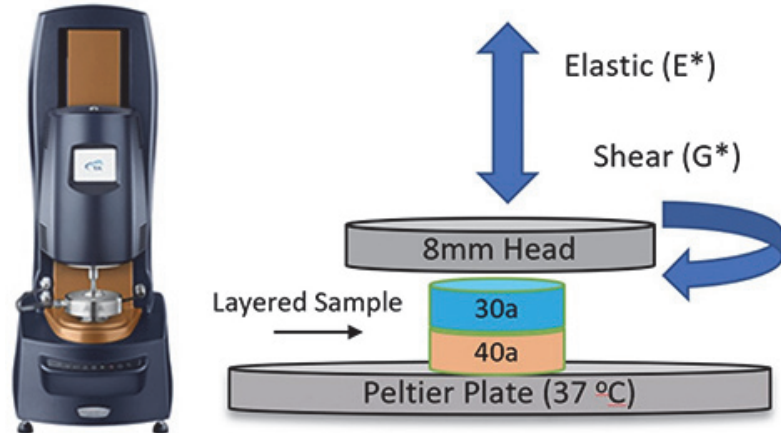

Abstract E-006 Figure 1 Left - Hybrid Rheometer. Right Rheometer Setup for Layered Sample

treatments. These models are also commonly used for the training of surgeons on innovate devices and new surgical procedures. These current vessel-training models cast from human vasculature with anatomical accuracy; however the materials often used for casting (i.e. silicone and glass) do not accurately simulate mechanical properties of tissue such as: vascular compliance (modulus), tensile and compressive strength, wall friction (lubricity), and hardness seen in native human vasculature. Thus, a more tunable and comprehensive in-vitro model material is needed to better understand how endovascular devices (i.e. microcatheters/wires, thrombectomy devices, coils, stents, flow diverters, and liquid embolics) interact with the vessel wall during delivery. An innovative 3D printed acrylic co-polymer (VeroClear ${ }^{\circledR}$ Agilus $30^{\circledR}-$ VCA30) can be tuned to match the mechanical properties of human vessels.

Materials and Methods Differing hardnesses of VC-A30 are layered and mechanically characterized with a hybrid rheometer (DHR 2, TA Instruments). The results are compared to replicated test results of donated 'fresh' human cadaveric tissue samples (Common Carotid Artery). Via the rheometer, data is collected for luminal wall friction, radial compliance, shear modulus, and elastic modulus (figure 1). Properties of cadaveric vessels and model materials are statistically compared to the VC-A30. The biomaterial layering is altered by varying the thickness of the VC-A30 layer thicknesses (biomaterial tuning) to mimic the mechanical properties of the cadaveric vasculature within statistical equivalence. Once successfully optimized, the biomaterials are manufactured into flow models and will additionally be validated by partnered neurointerventionalists to ensure that the model has realistic catheter trackability and is anatomically accurate.

Results The VC-A30 materials in the previous studies simulated the compliance and mechanical properties of human vasculature more closely than existing in-vitro silicone materials. VC-A30 is 3D printed to achieve accurate anatomical features; moreover, this $3 \mathrm{D}$ printed material is layered to simulate the lubricious inner lumen of a vessel, with varying hardness profiles to mimic vessel compliance and strength. This assembled structure simulates the layers of human vasculature and its variable properties.

Conclusion The utilization of novel biomimetic materials within this in-vitro vascular flow model will allow for more relevant benchtop testing of endovascular devices. These models have the potential to generate more accurate data on device performance and may reduce the need for costly in-vivo studies. 